\title{
Geschlechtsuntypische Laufbahnpläne - Wie interessant ist der Lehrerberuf für Gymnasiasten?
}

\section{Simone Berweger, Christa Kappler, Andrea Keck Frei und Christine Bieri Buschor}

\begin{abstract}
Der vorliegende Beitrag geht der Frage nach, inwiefern sich 16-jährige Gymnasiasten für den Lehrerberuf interessieren und welche Faktoren die Absicht beeinflussen, später ein Lehramtsstudium aufzunehmen. Die schriftliche Befragung an Zürcher Gymnasien zeigt, dass junge Männer $(n=388)$ bereits zu Beginn der Berufsorientierung ein geringes Interesse am Lehrerberuf aufweisen. Befunde einer sequenziellen, binär logistischen Regressionsanalyse ergaben, dass die Absicht, den Lehrerberuf zu ergreifen, vom Interesse und dem Wunsch nach Vereinbarkeit von Beruf und einer zeitintensiven Zusatzbeschäftigung abhängig ist, sowie die Bedeutung eines guten Einkommens als auch der Möglichkeit zu Teilzeitarbeit einen negativen Einfluss haben.
\end{abstract}

\section{Einleitung}

Viele Berufe und Sektoren sind geschlechtssegregiert, wobei Berufe mit einem höheren Männeranteil als prestigeträchtiger gelten (Leuze \& Strauss, 2009). Die Übervertretung von Männern bzw. Frauen geht häufig mit stereotypen Bildern von «typisch» männlichen und weiblichen Fähigkeiten und Interessen einher und schlägt sich in der Studien- und Berufswahl nieder. Dies lässt sich auch für den Lehrerberuf beobachten, in dem Frauen weltweit in der Mehrzahl sind (UNESCO, 2011). Während der Lehrerberuf in der Schweiz lange ein typischer Männerberuf war, wird seit den 1960er Jahren von einer, oft negativ konnotierten, «Feminisierung» des Volksschullehrerberufs gesprochen (Bloch Pfister, 2007). Vom Geschlecht der Lehrperson wird auf einen geschlechtsspezifisch ausgerichteten Unterricht geschlossen, der sich - so die Befürchtungen - besonders bei Lehrerinnen nachteilig auf die Persönlichkeitsentwicklung und den Schulerfolg von Jungen auswirke. Dadurch haben sich die Erwartungen an männliche Lehrpersonen stärker akzentuiert (Hannover \& Kessels, 2011; Skelton, 2012). So wird etwa gefordert, Männer müssten die Interessen der Jungen stärker in den Unterricht einbringen und als Rollenmodelle fungieren. 
Gleichzeitig werden ihnen häufig "weibliche» Fähigkeiten wie Einfühlungsvermögen abgesprochen (Brownhill, 2014). Die geschlechtsrollenstereotype Debatte, die in den letzten Jahren insbesondere in Westeuropa, den USA und Australien geführt wurde, dürfte zu einer Verunsicherung der (angehenden) Lehrpersonen beigetragen haben (Hutchings, Carrington, Skelton, Read \& Hall, 2007; Skelton, 2012).

Bislang sind die Zusammenhänge zwischen dem Geschlecht der Lehrperson und der Entwicklung der Schüler/innen wenig belegt, es bestehen keine Hinweise für eine mangelnde professionelle Haltung von Lehrerinnen (Brownhill, 2014; Horstkemper, 2010). Ein ausgeglicheneres Geschlechterverhältnis wäre jedoch unter dem Aspekt des Diversity Managements sinnvoll. Für Chancengleichheit bei der Studien- und Berufswahl sollten nicht geschlechtsstereotype Vorstellungen, sondern die Umsetzung des individuellen Potentials jenseits des «Geschlechterdenkens» ermöglicht werden. Analog zu den von Brentano (1963, zit. in Beaufaÿs, 2003) formulierten Leitlinien zur Untersuchung der geringen Beteiligung von Frauen in der Wissenschaft stellt sich die Frage: Können, sollen oder wollen junge Männer (nicht) Lehrer werden? Das «Können» (1) bezieht sich auf die Kompetenzen sowie auf die subjektive Einschätzung der eigenen Fähigkeiten. Mit dem «Sollen» (2) wird der Einfluss gesellschaftlich bedingter Normvorstellungen, Zuschreibungsmechanismen und Strukturbedingungen aufgegriffen. Das "Wollen» (3) verweist auf motivationale Bedingungen, Interessen sowie die unterschiedliche Priorisierung vorhandener Ziele von Individuen. Im Zentrum des vorliegenden Artikels stehen zentrale Faktoren einer handlungstheoretisch orientierten Sicht auf den Entscheidungsprozess im Laufe der Berufsorientierung: das Interesse junger Männer am Lehrerberuf und die Absicht, das Lehramtsstudium bzw. den Lehrerberuf zu wählen. Die Berufsorientierung ist an den Schweizer Gymnasien bis heute nicht gesetzlich verankert und daher den Jugendlichen, ihren Eltern sowie besonders engagierten Lehrkräften überlassen. Die Exploration und Schärfung der eigenen fachlichen Interessen und Fähigkeiten wird früh angeregt, indem sich Gymnasiast/innen der deutschsprachigen Schweiz mit rund 15 Jahren für eines von sechs Profilen entscheiden ${ }^{1}$. Allerdings gibt es kein Profil, das sich speziell für die Studienwahl Lehramt eignet. Umso dringlicher stellt sich die Frage nach den Faktoren, die die Wahl des Lehramtsstudiums begünstigen.

\section{Theorie und Forschungsstand}

\section{Theoretische Ansätze zur Erklärung der Studien- und Berufswahl}

Vor dem Hintergrund von Berufs- und Laufbahnmodellen, die den Berufs- und Studienwahlprozess als Durchlaufen von idealtypischen Phasen beschreiben (bspw. Heinz, 1984) ist davon auszugehen, dass sich 16-jährige Jugendliche in der 
Konkretisierungsphase am Anfang der Berufsorientierung befinden, in der diffuse Berufsbilder geschärft und mit Informationen aus der Berufswelt abgeglichen werden. Bis zu diesem Zeitpunkt hat gemäss Gottfredson $(1981,2002)$ bereits eine Eingrenzung individuell wählbarer Berufe stattgefunden: Zuerst werden Berufe ausgeschlossen, die als geschlechtsuntypisch wahrgenommen werden, danach solche, die bezüglich Sozialprestige als nicht passend erscheinen, und schliesslich im Jugendalter zunehmend jene, die nicht den eigenen Interessen und Wertvorstellungen entsprechen. Das Ergebnis dieser Eingrenzung ist eine "Zone akzeptabler Berufe», in der nach kongruenten Berufsfeldern und Tätigkeiten gesucht wird (vgl. Ratschinski, 2009). Dabei ist aufgrund des Einflusses der sozialen Umwelt anzunehmen, dass diejenigen Gymnasiast/innen, die sich (immer noch) für den Lehrerberuf interessieren, wesentlich durch (frühe) Lernerfahrungen und durch geeignete Rollenmodelle beeinflusst wurden, die den Lehrerberuf als wertvollen und für beide Geschlechter wählbaren Beruf darstellen. Die Jugendlichen, die ihre beruflichen Ziele mit der antizipierten Berufstätigkeit erfüllt sehen, dürften - trotz gesellschaftlich bedingter Vorstellungen bzgl. Geschlechtstypik und Prestige - den Lehrerberuf bei der Berufsexploration weiterhin berücksichtigen, im Hinblick auf eigene Interessen und Fähigkeiten prüfen und in den Prozess der Eingrenzung und Kompromissbildung (Gottfredson, 1981, 2002) einbeziehen. Die mit zunehmendem Alter immer stärkere Eingrenzung der «Zone akzeptabler Berufe» wurde in verschiedenen Studien nachgewiesen (vgl. Ratschinski, 2009). Fragt man Jugendliche nach ihren Traumberufen als Kind (Herzog, Neuenschwander \& Wannack, 2004), so zeigt sich, dass Jungen den Lehrerberuf schon relativ früh als berufliche Option ausschliessen.

Die sozial-kognitive Laufbahntheorie (SCCT; vgl. Lent, Brown \& Hackett, 1994, 2000) fokussiert mit dem «Model of Career Choice» den Prozess der Entscheidungsfindung, der zur Wahl eines Berufs/Studiums führt. Das Modell erklärt Verhalten im Berufswahlprozess aufgrund sozial-kognitiver Faktoren (Erwartungen und Ziele), die das aktive Handeln sowohl direkt als auch indirekt über das Interesse beeinflussen. Die Berufs- und Studienwahlmotivation resultiert aus dem Zusammenspiel intrinsischer und extrinsischer Motive, die in den erwarteten Handlungsfolgen zum Ausdruck kommen, sowie aus den Selbstwirksamkeitserwartungen und dem Interesse («I like/dislike») an berufsbezogenen Aktivitäten und Tätigkeiten. Spezifisch auf die Erklärung der Berufswahl Lehrer/in bezieht sich das «FIT-Choice»-Modell (Richardson \& Watt, 2006). Es berücksichtigt den Einfluss der Einschätzung der Anforderungen und des "task return" (sozialer Status, Lohn) sowie von Berufswahlmotiven, die sich auf den Wert und die Konsequenzen der Berufswahl Lehramt beziehen. Letztere umfassen die Einschätzung der eigenen Fähigkeit zu unterrichten, das Interesse am Lehrerberuf, die subjektive Bedeutung des persönlichen Nutzens sowie des sozialen Engagements als Lehrer/in, den Aspekt des Lehramts als Verlegenheitslösung, frühere Lehr- und Lern- bzw. Unterrichtserfahrungen sowie soziale 
Einflüsse (vgl. König, Rothland, Darge, Lünnemann \& Tachtsoglou, 2013). Die von Richardson und Watt (2006) erwähnte inhaltliche Nähe zur allgemeineren Berufswahlliteratur zeigt sich beim Vergleich mit der SCCT (Lent et al., 1994, 2000) darin, dass diese den Selbstwirksamkeitserwartungen, dem Interesse sowie den Handlungsfolgeerwartungen einen zentralen Stellenwert zuschreibt, und ebenfalls Lernerfahrungen sowie soziale Einflussfaktoren betont.

\section{Faktoren, die die Studienwahl "Lehrer" begünstigen}

Das Interesse und die Absicht 16-jähriger männlicher Jugendlicher an der Studien- und Berufswahl "Lehrer» sind in der Schweiz in dieser Altersgruppe bislang noch wenig untersucht. Eine Ausnahme bildet die Studie von Herzog et al. (2004), die auf geschlechtsspezifische Präferenzen hinweist und vermuten lässt, dass Jungen den Lehrerberuf früh als "Frauenberuf» wahrnehmen und daher ausschliessen. Dass jungen Männern in einer späteren Phase der Berufsorientierung bzw. am Übergang Gymnasium - Hochschule der Lehrerberuf im Vergleich zu anderen Berufs- und Studienoptionen weniger attraktiv scheint, wurde in einigen Schweizer Studien nachgewiesen (Bieri Buschor, Berweger, Keck Frei \& Kappler, 2012; Denzler \& Wolter, 2009; Denzler, 2014).

Studien zur geschlechtsuntypischen Studienwahl von Männern weisen darauf hin, dass dem Beruf der Eltern eine wichtige Rolle zukommt. Geschlechtsuntypische Berufs- und Studienwahlpläne bei 15-jährigen Männern hängen im Vergleich zu den Frauen deutlich stärker vom beruflichen Rollenmodell des Vaters ab, unabhängig vom sozio-ökonomischen Hintergrund (Helbig \& Leuze, 2012). Im Lehrerberuf ist die «Berufsvererbung» verglichen mit anderen Berufen überdurchschnittlich hoch, wobei sich dies für die Gymnasialstufe deutlicher zeigt als für die Volksschulstufe (Herzog, Herzog, Brunner \& Müller, 2007; Rothland, König \& Drahmann, 2015). Die verglichen mit anderen Studiengängen häufigere Herkunft aus einer Nichtakademikerfamilie zeigt sich nicht generell, sondern stufenspezifisch bei Lehramtsstudierenden der Sekundarstufe I (Keck Frei, Berweger, Denzler, Bieri Buschor \& Kappler, 2012).

Dass sich männliche Jugendliche für den Lehrerberuf interessieren, hängt wesentlich von ihren familiären und (ausser-)schulischen Lernerfahrungen ab (Kappler, 2013; Ulich, 2004), die sich in bereichsspezifischen Selbstwirksamkeitsüberzeugungen niederschlagen (Bandura, 1997). Erfahrungen aus Praktika oder dem Zivildienst dienen als «door opener» zu den als "weiblich» geltenden Berufen im pädagogischen und pflegerischen Bereich (Faulstich-Wieland, Niehaus \& Scholand, 2010; Tremel \& Möller, 2006). Nieskens (2009) zeigte, dass pädagogische Vorerfahrungen bereits während der gymnasialen Oberstufe in einem signifikanten Zusammenhang zu den lehrerberufsspezifischen Interessen stehen. Ob eine gymnasiale Profilwahl mit einem pädagogischen Schwerpunkt die Wahrscheinlichkeit für die Wahl des Lehramts erhöht, ist jedoch unklar (Denzler \& Wolter, 2009; Mombelli-Matthys, 2011).

Ländervergleichend haben sich bei den Berufswahlmotiven fünf Faktoren 
als besonders bedeutsam erwiesen: das Interesse an der Lehrtätigkeit («intrinsic value»), die subjektiv wahrgenommene Fähigkeit zu unterrichten, die Bedeutsamkeit des sozialen Engagements und der Zusammenarbeit mit Kindern und Jugendlichen sowie positive Lehr- und Lernerfahrungen (König et al., 2013; Rothland, 2011; Watt et al., 2012). In anderen Studien wird als Hauptmotiv die Freude am Umgang mit Kindern und Jugendlichen genannt, angehende Lehrer gewichten dieses Motiv jedoch weniger stark als Frauen (Herzog et al., 2007; Ulich, 2004). Zudem weisen sie im Vergleich mit Studenten anderer Fachgebiete eine geringere Karriereorientierung auf (Heine, Spangenberg, Schreiber \& Sommer, 2005). Nützlichkeitserwägungen, die sich u.a. auf ihre spätere Absicherung beziehen, spielen jedoch für ihren Berufsentscheid durchaus eine Rolle (Pohlmann \& Möller, 2010; Retelsdorf \& Möller, 2012). In der subjektiven Wahrnehmung von Studierenden wird die Entlohnung eher kritisch beurteilt, wobei Länderunterschiede mit realen Differenzen in den Lohnniveaus übereinstimmen (König et al., 2013; Richardson \& Watt, 2006). In der Schweiz ist der Einstiegslohn für Lehrpersonen vergleichsweise attraktiv, ab der mittleren Phase des Erwerbslebens jedoch verliert der Lehrerberuf für Männer an ökonomischem Nutzen, da sie in anderen Berufslaufbahnen höhere Löhne erzielen (Wolter, Denzler \& Weber, 2003). Wird die finanzielle Nützlichkeit hoch gewichtet, so steigt die Wahrscheinlichkeit, dass anstelle des Grundschullehramts das gymnasiale Lehramt gewählt wird (Retelsdorf \& Möller, 2012). Für Männer stellt der Lehrerberuf auch häufig ein «Sprungbrett» für eine andere Laufbahn oder eine Absicherung im Fall eines späteren beruflichen Misserfolgs dar (Herzog et al., 2007; Kappler, 2013; Watt \& Richardson, 2008). Im Vergleich zu Männern mit einer anderen Studienwahl messen Lehramtsstudenten der Gymnasialstufe der Vereinbarkeit von Familie und Beruf eine höhere Bedeutung zu (Abele, 2011). Der Lehrerberuf bietet Männern zudem die Möglichkeit, den Beruf mit ausserberuflichen Interessen wie z.B. Sport zu vereinbaren (Herzog et al., 2007; Mägdefrau, 2008; Pohlmann \& Möller, 2010; Schaarschmidt, 2004).

Bei der Analyse von Bedingungsfaktoren, die die (Nicht-)Wahl des Lehrerberufs beeinflussen, dominieren Studien, die Studienanfänger/innen nach Eintritt in die Ausbildung untersuchen und die Studien- und Berufswahl somit retrospektiv betrachten (Herzog et al. 2007; Retelsdorf \& Möller, 2012; Rothland, 2011). Vor dem Hintergrund theoretischer Überlegungen zur Prozesshaftigkeit der Berufs- und Studienwahl sowie der Frage, weshalb Männer den Beruf des Volksschullehrers nur noch selten ergreifen, drängten sich ein prospektiver Fokus sowie eine Eingrenzung der Stichprobe auf Gymnasiasten auf. Hier setzt die vorliegende Studie an: Sie geht der Frage nach, welche Faktoren wichtig sind, damit junge Männer zu Beginn der Berufsorientierung den Lehrerberuf überhaupt als Option wahrnehmen und dadurch in den Prozess der Eingrenzung und Kompromissbildung einbeziehen.

Vor dem Hintergrund dieser Ausführungen untersuchen wir im vorliegenden Beitrag folgende Fragestellungen: 
1. Wie stark ist das Interesse 16-jähriger Gymnasiasten für den Lehrerberuf verglichen mit anderen Fachrichtungen, und wie stark ist ihre Intention für ein Lehramtsstudium?

2. Inwiefern erklären familiäre Merkmale, Selbstwirksamkeitserwartungen, extrinsische Berufswahlmotive und das Interesse am Lehramt die Absicht, ein Lehramtsstudium zu ergreifen?

\section{Methodisches Vorgehen}

Die vorliegenden Ergebnisse stammen aus dem SNF-Forschungsprojekt "GUNST - Geschlechts(un)typische Studienwahl», das anhand mehrerer Teilstudien den Prozess der Berufs- und Studienwahl von Gymnasiast/innen ab der Profilwahl bis zwei Jahre nach Abschluss der Matura erforschte (Bieri Buschor et al., 2012).

\section{Stichprobe}

Im Frühjahr 2011 wurden 1080 Gymnasiast/innen aus zehn Gymnasien des Kantons Zürich während einer regulären Unterrichtslektion mittels Fragebogen zu fachlichen Interessen, Präferenzen im Hinblick auf die Berufs- und Studienwahl, Lernerfahrungen, Selbstwirksamkeitserwartungen und ihrer sozialen Herkunft befragt. Der Rücklauf betrug 86\% ( $\mathrm{n}=926)$. Die 388 Männer (42\%) und 537 Frauen (58\%) (eine Person ohne Geschlechtsangabe) besuchten das 9. Schuljahr und hatten neun Monate zuvor das gymnasiale Profil gewählt. Die Verteilung der Befragten auf die gymnasialen Profile weicht beim neusprachlichen und mathematisch-naturwissenschaftlichen Profil von der Gesamtverteilung im Kanton Zürich ab (+10\% bzw. -9\%) (Bildungsstatistik Kanton Zürich, 2014). Die Mehrheit der Befragten (81\%) war zum Zeitpunkt der Untersuchung 15-16 Jahre alt, der Anteil Ausländer/innen liegt bei 12\%. Rund die Hälfte stammt aus direkten Nachbarländern der Schweiz. Mehr als die Hälfte wurde im Ausland geboren (63\%), davon kamen 46\% vor Schulbeginn in die Schweiz. Ein Drittel der jungen Männer (36\%) hat mindestens einen Elternteil mit einer akademischen Ausbildung, für die Hälfte (51\%) trifft dies nicht zu (13\% fehlende Angaben). 13\% haben eine Mutter und 6\% einen Vater mit Lehrdiplom der Volksschule (9\% fehlende Angaben).

Bei etwas mehr als der Hälfte der jungen Männer $(58 \%, n=221)$ ist die Wahscheinlichkeit für ein Lehramtsstudium sehr gering ausgeprägt bzw. lässt eine «Null-Intention» bezüglich dieser Studienwahloption vermuten (0-20\%). Für die multivariaten Analysen wurden diese Männer nicht berücksichtigt, da vor dem Hintergrund der Theorie von Gottfredson (1981) davon ausgegangen werden muss, dass sie den Lehrerberuf aus der "Zone akzeptabler Berufe» (ebd.) bereits definitiv ausgeschlossen haben. Stattdessen wird der Fokus auf jene Gymnasiasten gerichtet, deren Studienintention darauf hinweist, dass 
das Lehramt bei der Exploration der Studienfächer zumindest am Rande des Feldes akzeptabler Alternativen als mögliche Option auftaucht. Nach Ausschluss von Personen mit fehlenden Angaben zur Ausbildung des Vaters konnte für die Modellierung eine Stichprobe von $\mathrm{n}=136$ (35\%) Gymnasiasten verwendet werden.

\section{Instrumente}

Interesse und Intention für die Wahl des Lehramtsstudiums der Volksschulstufe. Die Gymnasiasten wurden a) nach ihrem Interesse am Lehramt sowie an zwölf weiteren Fächergruppen gefragt ( $1=$ sehr gering bis $5=$ sehr stark) (vgl. Abb. 1), sowie b) um eine Einschätzung der Wahrscheinlichkeit für die Wahl dieser Studienfächer gebeten $(0-20 \%=$ fehlend bis sehr gering; $21-40 \%=$ eher gering; $41-60 \%=$ mittel; 61-80\%=eher hoch; $81-100 \%=$ sehr hoch) (vgl. Abb. 2).

Selbstwirksamkeitserwartungen. In Anlehnung an die Lehrer-Selbstwirksamkeits-Skala (Schwarzer \& Schmitz, 1999) wurde eine Skala (5 Items, Cronbach's Alpha=.82) zu Selbstwirksamkeitserwartungen im Lehrerberuf entwickelt, die sich auf gestalterisch-kreative und sportliche Aktivitäten mit Kindern und Jugendlichen bezieht (bspw. «Ich traue mir zu, mit Kindern eine Aufführung - z.B. Theater - einzustudieren»; $1=$ stimmt nicht bis $5=$ stimmt genau). Eine zweite Skala (6 Items, Cronbach's Alpha=.85) erfasst die fachlich-pädagogische Selbstwirksamkeit (bspw. «Ich traue mir zu, anspruchsvolle Inhalte von Schulfächern so aufzubereiten, dass sie für andere gut nachvollziehbar sind»; 1=stimmt nicht bis $5=$ stimmt genau).

Berufswahlmotive. Zwecks Vergleichbarkeit mit den anderen Teilstudien des Forschungsprojektes "GUNST» (Projektstart 2005) wurden die Faktoren zu Anreizen der Studien- bzw. Berufswahl breit und nicht spezifisch auf den Lehrerberuf bezogen erfasst. In Anlehnung an Fiechter, Stienen und Bühler (2004) wurde erhoben, wie wichtig den Gymnasiasten verschiedene extrinsische Anreize für die Wahl ihres künftigen Berufs sind (bspw. "Es ist mir besonders wichtig, ein gutes Einkommen zu erzielen»; 1 =trifft gar nicht zu bis $4=$ trifft völlig zu). Für die vorliegenden Analysen wurden vier Themenbereiche (Einzelitems) berücksichtigt, die in der Literatur zur Berufswahl Lehrer/in häufig genannt werden und empirische Relevanz aufweisen (Denzler \& Wolter, 2009; König et al., 2013; Richardson \& Watt, 2006; Rothland, 2011): (1) Einkommen bzw. monetärer Nutzen, (2) Möglichkeit zur Teilzeitarbeit, (3) Vereinbarkeit von Beruf und Familie sowie (4) Vereinbarkeit Beruf und zeitintensive(s) Hobby/ Zusatzbeschäftigung.

Ausbildung der Eltern bzw. des Vaters zur Volksschullehrperson. Die Gymnasiasten gaben Auskunft über die Bildungsabschlüsse ihrer Eltern. Zudem wurden sie in einer offenen Frage darum gebeten, eine möglichst genaue Berufsbezeichnung der Eltern anzugeben. Aufgrund der beiden Angaben wurde ein dummy-codierter Indikator gebildet, der Aufschluss darüber gibt, ob der Vater und/oder die Mutter ausgebildete/r Volksschullehrer/in ist, und ein weiterer Dummy zur Herkunft aus einer Akademikerfamilie. 


\section{Statistische Analyse}

Zur Beantwortung der ersten Forschungsfrage wurde über die gesamte Stichprobe $(n=926 / 925)$ hinweg das Interesse an 13 Fächergruppen sowie die Intention, ein Fachgebiet daraus zu wählen, untersucht. Bei der zweiten Forschungsfrage zur Vorhersage der intendierten Studienwahl Lehramt junger Männer wurde mit einer sequenziellen, binär logistischen Regressionsanalyse untersucht, anhand welcher von insgesamt 8 Prädiktoren sich Gymnasiasten, die eine eher geringe Intention für ein Lehramtsstudium aufweisen, von jenen mit einer mittleren bis sehr hohen Intention unterscheiden. Für eine bessere Interpretierbarkeit der Regressionsgewichte wurden sämtliche Prädiktorvariablen z-standardisiert. Die bivariaten Korrelationen zwischen den Prädiktoren fallen mit zwei Ausnahmen eher gering aus $(\mathrm{r}<.29) .^{2}$ Bei den deskriptiven Befunden wird die Stichprobe der Frauen zum Vergleich beigezogen.

Die in Tabelle 1 dargestellten Effekt-Koeffizienten $\exp \left[b_{j}\right]$, auch als «odds ratio» bezeichnet, geben Auskunft über die Wirkungsrichtung und -stärke der Prädiktoren. Ein Wert von 12.70 für die Variable Interesse für die Tätigkeit als Lehrer (Modell 3) besagt beispielsweise, dass sich bei Erhöhung des x-Wertes um eine Einheit das Chancenverhältnis $\mathrm{p}$ (Gruppe «eher geringe Intention für die Wahl des Lehramts»)/p(Gruppe «mittlere bis sehr hohe Intention») von ursprünglich 1:1 auf 12.70:1 verändert (Backhaus, Erichson, Plinke \& Weiber, 2008). In der vorliegenden Studie wurde der Pseudo $\mathrm{R}^{2}$-Koeffizient nach Nagelkerke (Pseudo $\mathrm{R}^{2} \mathrm{NK}$ ), der ab einem Wert von 0.5 als sehr gut gilt, sowie der Hosmer-Lemeshow-Test verwendet (akzeptabel ab p >.70), um die Variation der Regressionsmodelle zu quantifizieren (ebd.; Tabachnick \& Fidell, 2001). Der Vergleich der Güte der geprüften Modelle, die durch die stufenweise Aufnahme der Prädiktoren erweitert wurden, erfolgte anhand eines Vergleichs ( $\chi^{2}$-Testwert) der Unterschiede in der -2 Log Likelihood zwischen den Modellen (Tabachnick \& Fidell, 2001).

\section{Ergebnisse}

\section{Interesse am Lehramt}

Zur Frage, ob bereits ein spezifisches Studienfach anvisiert wurde, zeigt sich, dass die jungen Männer etwas weniger häufig $(n=168,44 \%)$ bereits eine solche Vorentscheidung getroffen haben als die Frauen $(\mathrm{n}=267,50 \%)\left[\chi^{2}(1)=3.845\right.$, $\mathrm{p}=.05]$. Insgesamt hat die Hälfte (53\%) noch kein konkretes Studienfach ins Auge gefasst, rund $14 \%$ sind noch unsicher, ob das Studienfach das richtige ist, ein Viertel ist sich eher sicher, und gut 7\% sind sich sehr sicher.

Die Gymnasiast/innen bewerteten 13 Fächergruppen nach Interesse und der Wahrscheinlichkeit, ein Studienfach daraus zu wählen (siehe Abb. 1). Lediglich $12 \%$ der Gymnasiasten geben ein eher oder sehr starkes Interesse für das Lehramt an, das damit die Option mit der geringsten Interessensbekundung ist. Auch 
die selbst eingeschätzte Wahrscheinlichkeit, ein Studium in einer bestimmten Fächergruppe zu ergreifen, ist beim Lehramt am geringsten: Nur 7\% bekunden eine eher oder sehr hohe Intention, ein Lehramtsstudium zu wählen (siehe Abb. 2). Sowohl beim Interesse als auch bei der Wahrscheinlichkeit für die Wahl eines spezifischen Studiums belegen die Naturwissenschaften sowie Wirtschaft und Recht die Spitzenpositionen.

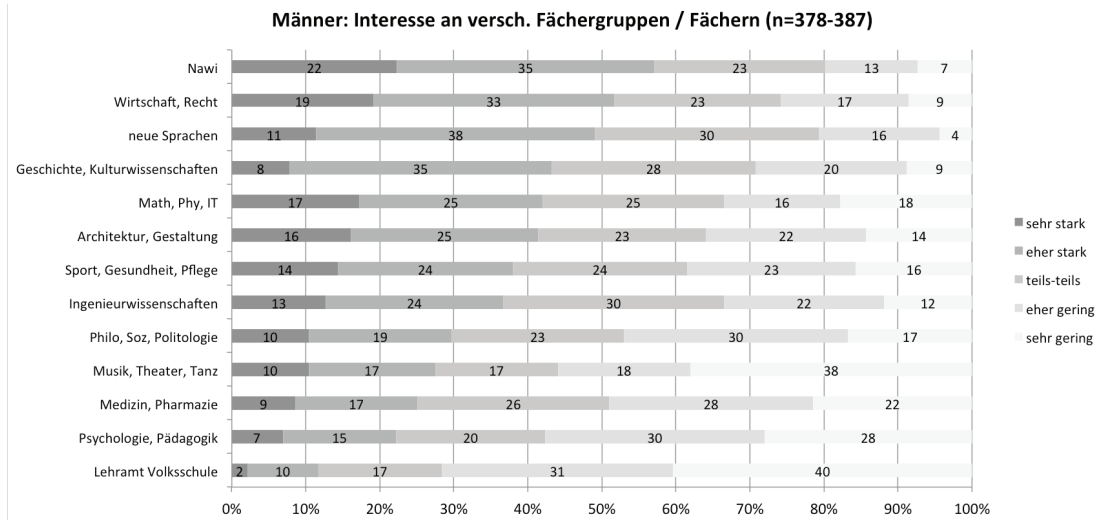

Abbildung 1: Interesse der Gymnasiasten an verschiedenen Fächergruppen/ Fächern ${ }^{3}$

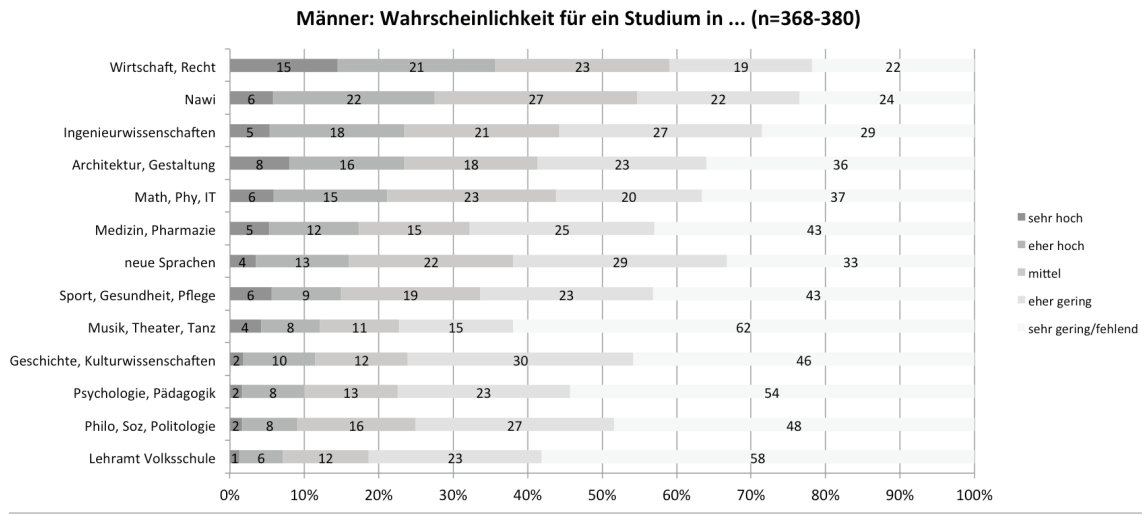

Abbildung 2: Wahrscheinlichkeit der Gymnasiasten für ein Studium in verschiedenen Fächergruppen

Der Vergleich mit den Gymnasiastinnen zeigt: Jede Dritte (33\%) bekundet ein eher oder sehr starkes Interesse für den Lehrerberuf, und jede Vierte $(25 \%)$ schätzt die Wahrscheinlichkeit, das Lehramtsstudium zu ergreifen, als hoch ein. Damit rangiert der Lehrerberuf bei den jungen Frauen unter den 13 Optionen auf Platz 10 bzw. 7. 
Beim Zusammenhang zwischen der Intention, das Lehramt zu wählen, und dem gymnasialen Profil zeigt sich, dass Männer mit einer mittleren bis sehr hohen Intention, verglichen mit den Altersgenossen mit einer eher geringen Intention sowie jenen mit einer "Null-Intention», nicht überzufällig häufig aus einem bestimmen Profil stammen $\left[\chi^{2}(8)=8.871, \mathrm{p}=.35\right]$. Auch zeigt sich kein Zusammenhang zwischen dem Profil und dem Interesse am Lehrerberuf $\left[\chi^{2}(16)=21.928, \mathrm{p}=.15\right]$.

Weder die soziale Herkunft noch ein Elternteil mit einer akademischen Ausbildung haben bei den jungen Männern einen Einfluss auf die intendierte Studienwahl Lehramt $\left[\chi^{2}(2)=1.274, p=.53 / \chi^{2}(2)=0.944, p=.19\right]$. Söhne von Volksschullehrern geben jedoch überzufällig häufig eine mittlere bis sehr hohe Intention für ein Lehramtsstudium an $\left[\chi^{2}(2)=9.294, p=.01\right]$.

\section{Absicht für ein Lehramtsstudium}

Mittels einer sequenziellen, binär logistischen Regressionsanalyse wurde anhand der Männerstichprobe untersucht, inwiefern die Wahrscheinlichkeit einer (Nicht-)Wahl des Lehramts durch einen Vater mit Lehramtsstudium, das Interesse am Lehramtsstudium auf Volksschulstufe, Selbstwirksamkeitserwartungen bezüglich der Arbeit mit Kindern und Jugendlichen sowie die Berufswahlmotive "Einkommen», "Teilzeitarbeit» und "Vereinbarkeit» erklärt werden kann. Bei der Reihenfolge der Aufnahme der Prädiktoren ins Modell (Metho$\mathrm{de}=$ ENTER) wurde in einem ersten Schritt das Merkmal zur «Berufsvererbung» (Vater mit Lehramtsstudium, dummy-codiert) ins Modell aufgenommen (Modell 1, M1), für das unter Kontrolle der anderen Prädiktoren eine Abschwächung im Effekt erwartet wurde. In einem zweiten Schritt wurde das Interesse am Lehramt berücksichtigt (M2), für das der stärkste Effekt erwartet und aufgrund theoretischer und empirischer Erwägungen vermutet wurde (bspw. Rothland et al., 2015), dass es den Effekt der Berufsvererbung abschwächen würde. Im dritten Schritt schliesslich wurden die Selbstwirksamkeitserwartungen sowie die Berufswahlmotive als Prädiktoren ins Modell aufgenommen (M3), für die ein weniger starker Effekt als für das Interesse angenommen wurde. Wie die Ergebnisse in Tabelle 1 zeigen, erhöht ein Vater mit Lehramtsstudium die Wahrscheinlichkeit, dass der Sohn den Lehrerberuf ernsthaft (d.h. mit einer mittleren bis sehr hohen Intention) in Betracht zieht, um ein 5- bzw. 6-faches (M1). Unter Kontrolle des Einflusses des Interesses am Lehramtsstudium (M2) verschwindet der Effekt der Berufsvererbung. Das Interesse erhöht die Wahrscheinlichkeit der Vorhersage der Gruppenzugehörigkeit um den Faktor 11.3. Weder die Erwartung, Fachinhalte gut vermitteln zu können, noch die Selbstwirksamkeit bezüglich kreativer und sportlicher Tätigkeiten im Lehrerberuf zeigen einen Effekt auf die Gruppenzugehörigkeit (M3). Bei den Berufswahlmotiven zeigt sich: Gymnasiasten, denen die Möglichkeit zur Teilzeitarbeit wichtig ist, schätzen die Wahrscheinlichkeit für ein Lehramtsstudium tiefer ein. Diejenigen, denen die Vereinbarkeit von Beruf und einer zeitintensiven Zusatzbeschäftigung (z.B. Sport) ein Anliegen 
ist, sowie jene, denen ein gutes Einkommen nicht so wichtig ist, befinden sich hingegen häufiger in der Gruppe mit einer mittleren bis sehr hohen Intention für den Lehrerberuf. Die Vereinbarkeit von Beruf und Familie hat keinen Effekt auf die Gruppenzugehörigkeit.

Tabelle 1: Binär logistische Regression zur intendierten Berufswahl "Lehrer»

\begin{tabular}{|c|c|c|c|c|c|c|c|c|c|c|c|c|}
\hline \multirow{3}{*}{$\begin{array}{l}\text { Modell } \\
\text { Prädiktoren }\end{array}$} & \multicolumn{12}{|c|}{$\begin{array}{l}\text { Wahrscheinlichkeit der Berufswahl «Lehrer» } \\
(0=\text { eher geringe, } 1=\text { mittlere bis sehr hohe Intention })\end{array}$} \\
\hline & \multicolumn{4}{|c|}{ M1 } & \multicolumn{4}{|c|}{ M2 } & \multicolumn{4}{|c|}{ M3 } \\
\hline & $b$ & $\mathrm{SE}_{b}$ & $p$ & $\begin{array}{l}\exp \\
{[\mathrm{b}]}\end{array}$ & $b$ & $\mathrm{SE}_{b}$ & $p$ & $\begin{array}{l}\exp \\
{[\mathrm{b}]}\end{array}$ & $b$ & $\mathrm{SE}_{b}$ & $p$ & $\begin{array}{l}\exp \\
{[\mathrm{b}]}\end{array}$ \\
\hline $\begin{array}{l}\text { Vater Lehrer vs. anderer } \\
\text { Beruf }\end{array}$ & 1.62 & .68 & .02 & 5.03 & 1.10 & .81 & .20 & 2.87 & 1.29 & .91 & .16 & 3.63 \\
\hline $\begin{array}{l}\text { Interesse am Lehramts- } \\
\text { studium }\end{array}$ & & & & & 2.42 & .42 & .00 & 11.27 & 2.54 & .48 & .00 & 12.70 \\
\hline \multicolumn{13}{|l|}{ Selbstwirksamkeit } \\
\hline $\begin{array}{l}\text { kreativ-gestalterische } \\
\text { Arbeit mit Kindern }\end{array}$ & & & & & & & & & .13 & .29 & .66 & 1.14 \\
\hline $\begin{array}{l}\text { fachliches Arbeiten } \\
\text { mit Kindern }\end{array}$ & & & & & & & & & .36 & .36 & .32 & 1.43 \\
\hline \multicolumn{13}{|l|}{ Berufswahlmotive } \\
\hline gutes Einkommen & & & & & & & & & -.55 & .28 & .05 & .58 \\
\hline Teilzeitarbeit & & & & & & & & & -.62 & .30 & .04 & .54 \\
\hline $\begin{array}{l}\text { Vereinbarkeit Beruf - } \\
\text { Familie }\end{array}$ & & & & & & & & & .38 & .32 & .24 & 1.47 \\
\hline $\begin{array}{l}\text { Vereinbarkeit Beruf- } \\
\text { zeitintensive Zusatz- } \\
\text { beschäftigung }\end{array}$ & & & & & & & & & .67 & .30 & .03 & 1.95 \\
\hline \multicolumn{13}{|l|}{ Modellstatistiken } \\
\hline$R^{2} \mathrm{NK}$ & .065 & & & & .562 & & & & .647 & & & \\
\hline$-2 * \ln (\mathrm{L})$ & 179.4 & & & & 112.3 & & & & 96.7 & & & \\
\hline \multicolumn{13}{|l|}{$\begin{array}{l}\text { Zuwachs an Erklärungs- } \\
\text { leistung }\end{array}$} \\
\hline Block $\chi^{2}$ & 6.704, & , $<<.05$ & & & 67.16 & $6, \mathrm{p}<$. & & & 15.59 & $6, \mathrm{p}<$. & & \\
\hline
\end{tabular}

Anmerkungen $R^{2} N K=R^{2}$ nach Nagelkerke; $S E_{b}=$ Standardfehler des Regressionskoeffizienten; $\operatorname{In}(L)=$ logarithmierte Likelihood des Modells.

Das vollständige Modell vermag mit insgesamt 8 Prädiktoren 65\% der Varianz in der abhängigen Variablen zu erklären (Nagelkerkes $\mathrm{R}^{2}$ ). Der Hosmer-Lemeshow-Test verweist mit $\mathrm{p}=.75$ auf eine akzeptable Zufallswahrscheinlichkeit (Backhaus et al., 2008; Tabachnick \& Fidell, 2001). Der Anteil der richtig klassifizierten Gymnasiasten liegt bei $81 \%$. 


\section{Diskussion}

Die Hälfte der 16-jährigen Gymnasiasten hat bereits ein konkretes Studienfach anvisiert - dies stützt die Annahme, dass sich die jungen Männer in der Phase der Konkretisierung von Wünschen und Vorstellungen bezüglich Ausbildung und Beruf befinden. Über die Hälfte gibt ein (eher) starkes Interesse für Naturwissenschaften und für Wirtschaft/Recht an. Das Interesse am Lehrerberuf ist mit 12\% Interessierten und 7\% mit einer hohen Wahrscheinlichkeit für eine entsprechende Studienwahl sehr gering, auch im Vergleich mit den jungen Frauen. Die "Zone akzeptabler Berufe» scheint wie vermutet (Gottfredson, 1981) bereits in dieser frühen Phase der Berufsorientierung auf mehrheitlich geschlechtstypische Fächergruppen eingegrenzt. Gut ein Viertel ist «eher sicher» im Hinblick auf die Wahl eines Studienfachs und 7\% berichten, schon «sehr sicher» und damit wohl nahe an einer Entscheidung zu sein.

Dem Interesse kommt erwartungsgemäss (Lent et al. 1994; Richardson \& Watt, 2006) der grösste positive Effekt auf eine eher geringe versus eine mittlere bis sehr hohe Absicht für die Wahl des Lehramtsstudiums zu, gefolgt vom Wunsch, den Beruf und eine zeitintensive Zusatzbeschäftigung gut vereinbaren zu können, was auch durch andere Studien gestützt wird (bspw. Schaarschmidt, 2004). Die hohe Bedeutung des Interesses an der Lehrtätigkeit im Sinne der «intrinsischen Motivation» wird auch in Studien zum FIT-Choice Modell (König \& Rothland, 2012; Watt et al., 2012) und mit dem FEMOLA (Pohlmann \& Möller, 2010) betont. Ein geringer negativer Effekt zeigt sich bei der «Wichtigkeit eines guten Einkommens» und der «Möglichkeit zur Teilzeitarbeit». Für die "Vereinbarkeit von Beruf und Familie» zeigt sich hingegen kein Effekt. Die schrittweise Modellierung macht deutlich, dass der Einfluss der Berufsvererbung (Vater ist Volksschullehrer) verschwindet, wenn das Interesse kontrolliert wird. Dies spricht in Übereinstimmung mit Rothland et al. (2015) dafür, dass diese Variable als ein distaler Umweltfaktor wirkt (Lent et al., 1994) und eine Lernumgebung begünstigt, die das Interesse am Lehrerberuf fördert. Ebenso ist der fehlende Effekt der Selbstwirksamkeitserwartungen möglicherweise darauf zurückzuführen, dass der Einfluss indirekt über das Interesse vermittelt wird (Lent et al., 1994), was in der vorliegenden Studie jedoch nicht untersucht wurde.

Die Ergebnisse zum Einfluss der Berufswahlmotive lassen sich dahingehend interpretieren, dass bei Gymnasiasten die Studienwahlintention "Lehrer» an den Nutzen für ihre private Lebensplanung gekoppelt ist, allerdings nicht in Zusammenhang mit der Vereinbarkeit von Beruf und Familie, sondern mit einer zeitintensiven Tätigkeit wie beispielsweise Sport. Im Vergleich zu Studien, die Nützlichkeitsaspekte bzgl. Familie und Freizeit (Retelsdorf \& Möller 2012; Watt \& Richardson, 2008) bzw. Familie und Teilzeitarbeit (Denzler \& Wolter, 2008) zusammenfassen, ermöglichen die vorliegenden Befunde eine differenzierte Sicht auf die Teilzeit-, Freizeit- und Familienorientierung von am Lehrer- 
beruf interessierten jungen Männern. Dass junge Männer den Lehrerberuf als berufliche Tätigkeit erachten, die sich auch bei einer Vollzeittätigkeit mit den ausserberuflichen Interessen optimal verbinden lässt, hat sich auch in anderen Studien gezeigt (Mägdefrau, 2008; Schaarschmidt, 2004).

Für die vorliegende Studie gelten einige Einschränkungen. Erstens wurden die im deutschsprachigen Raum vorhandenen Instrumente zur Analyse der Studienwahlmotivation (FIT-Choice-Modell und FEMOLA) nicht einbezogen, was die Vergleichbarkeit erschwert. Allerdings zeigen sich zahlreiche Ähnlichkeiten in den theoretischen Konstrukten. Zudem ist die Studie in einen theoretischen Bezugsrahmen eingebettet, der Parallelen zum «expectancy-value» Ansatz aufweist und der empirisch besonders bedeutsame Aspekte der Motivation für ein Lehramtsstudiums abdeckt (vgl. König et al. 2013). Zweitens ist die untersuchte Stichprobe relativ klein und erfüllt die Ansprüche an die Repräsentativität nur teilweise. Zudem lässt die Querschnittstudie keine Aussagen über Prozesse und Veränderungen bei der Studien- und Berufswahl zu. Drittens wurde die Frauenstichprobe nur partiell, d.h. im deskriptiven Teil, als Vergleichsgruppe beigezogen.

\section{Ausblick}

Angesichts des Resultats, dass einerseits die Hälfte der Gymnasiasten noch keine Studien- und Berufspräferenz bekundet und andererseits die bereits entschiedenen Männer eher geschlechtstypische Interessen und Absichten äussern, scheint eine gezielte Studienfachexploration bzw. Berufsorientierung sowie die Unterstützung des Entscheidungsfindungsprozesses - die sich in den letzten Jahren einige Kantone zum Ziel gesetzt haben - von hoher Bedeutung (z.B. Bildungsdirektion Kanton Zürich, 2014). Seit 2012 widmet sich ein EDK-Projekt der Verbesserung der Studien- und Laufbahnberatung an Gymnasien (EDK, 2013) mit dem Ziel, Studienabbrüche und Studienfachwechsel zu verringern. Die strukturierte Begleitung der Studien- und Berufswahl im Gymnasium ermöglicht Kontakte zu Rollenvorbildern und trägt zu einem realistischeren Bild der Studienfächer und der Berufswelt bei. Wie Nieskens (2009) für den Lehrerberuf aufzeigte, stellen sich selbst Schüler/innen, die ausgeprägte «Passungsmerkmale» (ebd., S. 254) aufweisen, den Berufsalltag einer Lehrperson überwiegend als langweilig und wenig anspruchsvoll vor. Insbesondere für eine Studien- und Berufswahl in Bereichen, in der die Geschlechterverteilung sehr unausgeglichen ist, sind die soziale Unterstützung von Lernerfahrungen und eine gezielte Exploration von Interessen und Studienwünschen aller Jugendlicher von hoher Bedeutung (Fock, Glumpler, Hochfeld \& Weber-Klaus, 2001; Lent et al., 1994).

Können, sollen oder wollen junge Männer nicht Volksschullehrer werden? Der vorliegende Beitrag zeigt, dass das «Wollen» Schwierigkeiten bereitet, denn der Anteil Gymnasiasten, der sich für das Lehramt interessiert und diese Studi- 
enwahl ernsthaft in Betracht zieht, ist insgesamt sehr gering. Ob dies durch die ambivalente Debatte um Männer im Lehrerberuf beeinflusst wird, die das «Sollen» zur Diskussion stellt, lässt sich durch die vorliegende Untersuchung nicht beantworten. Werden das Interesse am Beruf bzw. den Fächern sowie die Intention für die Wahl derselben betrachtet, scheint der Lehrerberuf im Vergleich zu anderen Laufbahnoptionen als unattraktiver wahrgenommen zu werden. Positiv beeinflusst wird das «Wollen» offenbar indirekt durch Väter, die selbst als Rollenmodelle fungieren und ein Umfeld für Lernerfahrungen bieten, das dem Interesse am Lehrerberuf förderlich ist. Das Bild des «vollzeittätigen» Lehrers, der Abstriche bei der Entlohnung in Kauf nimmt, und mit der Berufswahl eine gute Vereinbarkeit von Beruf und zeitintensiven Hobbies zu realisieren sucht, bietet spannende Anknüpfungspunkte für weitere Studien. Über die Dynamik im Zusammenhang mit der Feminisierung kann an dieser Stelle nur spekuliert werden. Wünschenswert sind Untersuchungen, die sich der Frage widmen, inwiefern sich veränderte Rollenerwartungen an Volksschullehrpersonen (breiter Berufsauftrag über das Kerngeschäft «Unterricht» hinaus) negativ auf das «Können» und "Sollen» auswirken. Es ist zu vermuten, dass junge Männer die «fürsorglich-erzieherischen» Aspekte als wenig geschlechtskonform wahrnehmen. Die stark wirkenden gesellschaftlich geprägten Leitbilder hinsichtlich der Berufswahl - das «Sollen» wie auch das "Können» - müssten durch Vorbilder bzw. Identifikationsfiguren auch ausserhalb der Familie überformt werden, damit sich Gymnasiasten überhaupt für den Lehrerberuf interessieren können. Eine Erhöhung des Männeranteils im Lehrerberuf dürfte ohne Veränderungen dieser Leitbilder und institutioneller Mechanismen wie etwa die stärkere Durchlässigkeit beruflicher Laufbahnen in den Lehrerberuf (Quereinstieg) und aus dem Lehrerberuf (Um- und Aufstieg) kaum realisierbar sein.

\section{Anmerkungen:}

1 Vor Beginn des Kurzzeitgymnasiums bzw. des dritten Jahres des Langzeitgymnasiums wählen die Schüler/innen ein Profil, das den weiteren fachlichen Schwerpunkt vorgibt. Als Profile zur Verfügung stehen im Kanton Zürich: altsprachlich, musisch, mathematisch-naturwissenschaftlich, neusprachlich, wirtschaftlich-rechtlich sowie «Philosophie, Pädagogik, Psychologie».

2 Ein hoher bzw. mittlerer Korrelationswert zeigt sich zwischen dem Interesse am Lehrerberuf und der Wahrscheinlichkeit eines Lehramtsstudiums ( $\mathrm{r}=.66)$ bzw. zwischen der Selbstwirksamkeit bezüglich der fachlichen Arbeit mit Kindern und der Selbstwirksamkeit bezüglich gestalterisch-kreativer Tätigkeiten $(\mathrm{r}=.45)$.

3 Aufgrund der Abweichung der Stichprobe von der Grundgesamtheit (Übervertretung neusprachliches Profil, Untervertretung math.-naturw. Profil) ist anzunehmen, dass in der Grundgesamtheit die Interessensbekundung für Naturwissenschaften, Mathematik/Physik/IT und Ingenieurwissenschaften eher höher, bzw. für neue Sprachen eher tiefer ausfällt. Das Interesse an neuen Sprachen ist bei den befragten Männern aus dem neusprachlichen Profil überzufällig hoch $\left[\chi^{2}(16)=36.085, p=.00\right]$, und Männer aus dem mathematisch-naturwissenschaftlichen Profil haben ein besonders hohes Interesse an Naturwissenschaften $\left[\chi^{2}(16)=54.827, \mathrm{p}=.00\right]$ sowie an Mathematik/Physik/IT 
$\left[\chi^{2}(16)=56.714, p=.00\right]$. Diese Ergebnisse zeigen sich ebenso beim Zusammenhang zwischen Profil und intendierter Studienwahl.

\section{Literatur}

Abele, A. E. (2011). Prädiktoren des Berufserfolgs von Lehrkräften. Zeitschrift für Pädagogik, 57(5), 674-694.

Backhaus, K., Erichson, B., Plinke, W. \& Weiber, R. (2008). Multivariate Analysemethoden. Eine anwendungsorientierte Einführung (12 ed.). Berlin: Springer.

Bandura, A. (1997). Self-Efficacy. The exercise of control. New York: Freeman.

Beaufaÿs, S. (2003). Wie werden Wissenschaftler gemacht? Beobachtungen zur wechselseitigen Konstitution von Geschlecht und Wissenschaft. Bielefeld: transcript.

Bieri Buschor, C., Berweger, S., Keck Frei, A. \& Kappler, C. (2012). "Geschlechts(un)typische» Studienwahl: Weshalb Frauen Ingenieurwissenschaften studieren und Männer Primarlehrer werden. Projektbericht. Pädagogische Hochschule Zürich.

Bildungsdirektion Kanton Zürich (2014). Unterstützung bei der Studienwahl an den Zürcher Mittelschulen. Zürich: Bildungsplanung.

Bildungsstatistik Kanton Zürich (2014). Mittelschulen: Abschlüsse nach Profil. Zugriff am 30.04.2015 unter http://www.bista.zh.ch/ms/Abschluss.aspx

Bloch Pfister, A. (2007). Priester der Volksbildung. Der Professionalisierungsprozess der Zürcher Volksschullehrkräfte zwischen 1770 und 1914. Zürich: Chronos.

Brownhill, S. (2014). «Build me a male role model!» A critical exploration of the perceived qualities/characteristics of men in the early years (0-8) in England. Gender and Education, 26(3), 246-261.

Denzler, S. (2014). Integration of Teacher Education into the Swiss Higher Education System. PhD Thesis, University of Lausanne.

Denzler, S. \& Wolter S. C. (2009). Sorting into teacher education: how the institutional setting matters. Cambridge Journal of Education, 39(4), 423-441.

Denzler, S. \& Wolter, S. C. (2008). Selbstselektion bei der Wahl eines Lehramtsstudiums: Zum Zusammenspiel individueller und institutioneller Faktoren. Beiträge zur Hochschulforschung, 30(4), 112-141.

EDK (2013). Gymnasiale Maturität: Langfristige Sicherung des prüfungsfreien Hochschulzugangs. EDK. Zugriff am 30.04.2015 unter http://www.edudoc.ch/static/web/arbeiten/ gymnasiale_maturitaet_d.pdf

Faulstich-Wieland, H., Niehaus, I. \& Scholand, B. (2010). Lehramt Grundschule: «niedrigste Stufe dieses Lehrerberufs» versus «ich liebe Kinder». Oder: Was SchülerInnen vom Lehramt abhält und Studierende daran reizt. Erziehungswissenschaft 2, 41, 27-42.

Fiechter, U., Stienen, A. \& Bühler, C. (2004). Zukünftige Lehrpersonen: Berufswahl als pragmatisch orientierte Individualisierung. Eine Studie zur Situation im Kanton Bern. Bern: Institut für Lehrerinnen- und Lehrerbildung.

Fock, C., Glumpler, E., Hochfeld, I. \& Weber-Klaus, S. (2001). Studienwahl: Lehramt Primarstufe. In E. Glumpler \& C. Fock (Hrsg.), Frauen in pädagogischen Berufen: Lehrerinnen (S. 212-240). Bad Heilbrunn: Klinkhardt.

Gottfredson, L. S. (2002). Gottfredson's theory of circumscription, compromise, and selfcreation. In D. Brown (Ed.), Career choice and development (4th ed.) (p. 85-148). San Francisco: Jossey-Bass.

Gottfredson, L. S. (1981). Circumscription and Compromise: A Developmental Theory of Occupational Aspirations. Journal of Counseling Psychology Monograph, 28(6), 545-579.

Hannover, B. \& Kessels, U. (2011). Sind Jungen die neuen Bildungsverlierer? Empirische Evidenz für Geschlechterdisparitäten zuungunsten von Jungen und Erklärungsansätze. Zeitschrift für Pädagogische Psychologie, 25(2), 89-103.

Heine, C., Spangenberg, H., Schreiber, J. \& Sommer, D. (2005). Studienanfänger 2003/04 
und 2004/05. Bildungswege, Motive der Studienentscheidung und Gründe der Hochschulwahl. Kurzinformation A 15/2005 des HIS. Hannover: HIS.

Heinz, W. R. (1984). Der Übergang von der Schule in den Beruf als Selbstsozialisation. Bremen: Universität.

Helbig, M. \& Leuze, K. (2012). Ich will Feuerwehrmann werden! Wie Eltern, individuelle Leistungen und schulische Fördermassnahmen geschlechts(un-)typische Berufsaspirationen prägen. Kölner Zeitschrift für Soziologie und Sozialpsychologie 64(1), 9-122.

Herzog, W., Herzog, S., Brunner, A. \& Müller, H. P. (2007). Einmal Lehrer, immer Lehrer? Eine vergleichende Untersuchung der Berufskarrieren von (ehemaligen) Lehrpersonen. Bern: Haupt.

Herzog, W., Neuenschwander, M. P. \& Wannack, E. (2004). In engen Bahnen: Berufswahlprozess bei Jugendlichen. Bern: Schweizerischer Nationalfonds.

Horstkemper, M. (2010). Frauen und Männer im Lehrerberuf. Geschlechterdifferenz und Professionalisierung. Friedrich Jahresheft, 50-53.

Hutchings, M. B., Carrington, C., Skelton, C., Read, B. \& Hall, I. (2007). Nice and kind, smart and funny: what children want to emulate in their teachers. Oxford Review of Education, 34(2), 135-157.

Kappler, C. (2013). Berufswahlprozesse und Motive angehender Lehrer. Eine qualitative Studie aus geschlechter- und berufsbiographisch-vergleichender Perspektive. Bern: Haupt.

Keck Frei, A., Berweger, S., Denzler, S., Bieri Buschor, C. \& Kappler, C. (2012). Wer selektioniert sich in die Ausbildung zur Sekundarlehrperson? Schweizerische Zeitschrift für Bildungswissenschaften, 34(3), 483-499.

König, J., Rothland, M., Darge, K., Lünnemann, M. \& Tachtsoglou, S. (2013). Erfassung und Struktur berufswahlrelevanter Faktoren für die Lehrerausbildung und den Lehrerberuf in Deutschland, Österreich und der Schweiz. Zeitschrift für Erziehungswissenschaft, 16(3), 553-577.

König, J. \& Rothland, M. (2012). Motivations for choosing teaching as a career: Effects on general pedagogical knowledge during initial teacher education. Asia-Pacific Journal of Teacher Education, 40, 289-315.

Lent, R. W., Brown, S. D. \& Hackett, G. (1994). Toward a unifying social cognitive theory of career and academic interest, choice and performance. Journal of Vocational Behaviour, 45, 79-122. Lent, R. W., Hackett, G. \& Brown, S. D. (2000). Contextual supports and barriers to career choice: A social cognitive analysis. Journal of Counseling Psychology, 47(1), 36-49.

Leuze, K. \& Strauss, S. (2009). Lohnungleichheiten zwischen Akademikerinnen und Akademikern: Der Einfluss von fachlicher Spezialisierung, frauendominierten Fächern und beruflicher Segregation. Zeitschrift für Soziologie, 38(4), 262-282.

Mägdefrau, J. (2008). Welche Werte haben zukünftige Lehrer/-innen? - Lehramtsstudierende und Studierende nicht pädagogischer Fachrichtungen im Vergleich. Zeitschrift für Soziologie der Erziehung und Sozialisation, 28(1), 36-55.

Mombelli-Matthys, D. (2011). Schwerpunktfach Philosophie/Pädagogik/Psychologie. Genese, Implementierung und Status quo eines neuen Unterrichtsfachs an Schweizer Gymnasien. Dissertation an der Universität Bern: Selbstverlag.

Nieskens, B. (2009). Wer interessiert sich für den Lehrerberuf-und wer nicht? Berufswahl im Spannungsfeld von subjektiver und objektiver Passung. Göttingen: Cuvillier.

Pohlmann, B. \& Möller, J. (2010). Fragebogen zur Erfassung der Motivation für die Wahl des Lehramtsstudiums (FEMOLA). Zeitschrift für Pädagogische Psychologie, 24, 73-84.

Ratschinski, G. (2009). Selbstkonzept und Berufswahl. Eine Überprüfung der Berufswahltheorie von Gottfredson an Sekundarschülern. Münster: Waxmann.

Retelsdorf, J. \& Möller, J. (2012). Grundschule oder Gymnasium? Zur Motivation ein Lehramt zu studieren. Zeitschrift für Pädagogische Psychologie, 26(1), 1-7.

Richardson, P. W. \& Watt, H. M. G. (2006). Who Chooses Teaching and Why? Profiling 
Characteristics and Motivations Across Three Australian Universities. Asia-Pacific Journal of Teacher Education, 34(1), 27-56.

Rothland, M., König, J. \& Drahmann, M. (2015). Lehrerkinder - Zur Bedeutung der Berufsvererbung für die Berufswahl Lehramt. Zeitschrift für Bildungsforschung (online). Wiesbaden: Springer Fachmedien.

Rothland, M. (2011). Warum entscheiden sich Studierende für den Lehrerberuf? In E. Terhart, H. Bennewitz \& M. Rothland (Hrsg.), Handbuch der Forschung zum Lehrerberuf (S. 268-295). Münster: Waxmann.

Schaarschmidt, U. (Hrsg.) (2004). Halbtagsjobber? Psychische Gesundheit im Lehrerberuf Analyse eines veränderungsbedürftigen Zustandes (2. Aufl.). Weinheim: Beltz.

Schwarzer, R. \& Schmitz, G. S. (1999). Kollektive Selbstwirksamkeitserwartung von Lehrern. Eine Längsschnittstudie in zehn Bundesländern. Zeitschrift für Sozialpsychologie, 30(4), 262-274.

Skelton, C. (2012). Men teachers and the "feminised" primary school: a review of the literature. Educational Review, 64, 1-19.

Tabachnick, B. \& Fidell, L. S. (2001). Using multivariate statistics (4 ed.). Boston: Allyn and Bacon.

Tremel, I. \& Möller, S. (2006). «Wenn es den Zivildienst nicht gäbe, würde es hier noch mal ganz anders aussehen...» Erste Forschungsergebnisse zu den Beweggründen junger Männer zur Aufnahme eines sonderpädagogischen / rehabilitationswissenschaftlichen Studiums. Journal Netzwerk Frauenforschung NRW, 20, 50-57.

Ulich, K. (2004). "Ich will Lehrer/in werden». Eine Untersuchung zu den Berufsmotiven von Studierenden. Weinheim: Beltz.

UNESCO (2011). The Global Demand for Primary Teachers - 2011 Update. Zugriff am 30.04.2015 unter http://www.uis.unesco.org/Education/Documents/IS6-2011-Teachers-EN6.pdf

Watt, H. M. G., Richardson, P. W., Klusmann, U., Kunter, M., Beyer, B., Trautwein, U. \& Baumert J. (2012). Motivations for choosing teaching as a career: An international comparison using the FIT-Choice scale. Teaching and Teacher Education, 28(6), 791-805.

Watt, H. M. G. \& Richardson P. W. (2008). Motivations, perceptions, and aspirations concerning teaching as a career for different types of beginning teachers. Learning and Instruction, 18(5), 408-428.

Wolter, S. C., Denzler, S. \& Weber, B. A. (2003). Betrachtungen zum Arbeitsmarkt der Lehrer in der Schweiz. Vierteljahrshefte zur Wirtschaftsforschung, 72(2), 305-319.

Schlagworte: Geschlechts(un)typische Studien- und Berufswahl, frühe Berufsorientierung, Gymnasiasten, Interesse am Lehrerberuf, Berufswahlmotive 


\section{Plans de carrières atypiques - Quel est l'intérêt des gymnasiens pour le métier d'enseignant?}

\section{Résumé}

Le présent article étudie l'intérêt porté par les gymnasiens de 16 ans au métier d'enseignant ainsi que les facteurs qui influencent ultérieurement la décision de suivre une formation pédagogique. L'enquête réalisée dans des gymnases zurichois démontre un faible intérêt des jeunes gens $(n=338)$ pour la carrière d'enseignant dès leur première orientation professionnelle. Les résultats, obtenus par une analyse de régression logistique séquentielle binaire, montrent que l'intention d'embrasser une carrière d'enseignant est influencée par l'intérêt pour l'enseignement et la motivation à concilier vie privée et professionnelle en maintenant une occupation extraprofessionnelle conséquente. L'opportunité d'un bon salaire et la possibilité d'exercer un emploi à temps partiel influencent négativement le choix d'une carrière d'enseignant.

Mots-clés: Études et choix professionnels (a)typiques pour un genre, orientation professionnelle précoce, gymnasiens, intérêt pour les études / la profession d'enseignant, motivations dans le choix de la profession

\section{Carriere atipiche per il gender - Quanto è interessante la professione docente per i liceali?}

\section{Riassunto}

Questo articolo analizza l'interesse dei giovani liceali di 16 anni per la professione docente e i fattori che influenzano la scelta o meno di intraprendere una formazione pedagogica ulteriore. Il questionario scritto somministrato nei licei zurighesi testimonia un interesse scarso da parte dei ragazzi $(n=338)$ per una carriera nell'insegnamento già all'inizio della fase di orientamento professionale. I risultati ottenuti attraverso un'analisi di regressione sequenziale logistica mostrano che l'intenzione di avviarsi o meno alla professione docente è influenzata dall'interesse per l'insegnamento e da motivazioni quali il desiderio di conciliare vita professionale e privata o di mantenere un impegno extra professionale. Influiscono anche - se pur in maniera negativa - la possibilità di lavorare a tempo parziale e di avere un buon salario.

Parole chiave: Scelte di studio e professionali atipiche rispetto al gender, orientamento professionale precoce, liceali, interesse per la professione docente, motivazione nella scelta professionale 


\section{Gender-typed career plans - To what extent are male high school students interested in teaching?}

\section{Summary}

This paper investigates to what extent 16-year old Swiss male high school students are interested in a teaching professional career. It analyses factors contributing to the intention to choose teaching after graduating from academic high school. The results of a survey $(n=388)$, conducted in the canton of Zurich, show that male adolescents report low interest in teaching at an early stage of their career orientation. Results of a sequential logistic regression analysis reveal a number of factors that are relevant to the participants' intention to opt for teaching as a career. Interest in teaching and a good work-life balance, including hobbies such as sports, have a positive effect, while considerations regarding financial security and opportunities to work part-time, in contrast, have a negative impact on opting for choosing the field of teaching.

Keywords: Gender-typed career plans, early stage of career orientation, male high school students, interest in teaching, factors related to career choice 
